\title{
CB Research Square \\ Toxicity of Perfluoroalkyl Substances (PFAS) Toward Embryonic Stages of Mahi-Mahi (Coryphaena Hippurus)
}

\author{
Kiflom Y. Gebreab \\ Florida International University \\ Daniel Benetti \\ University of Miami, \\ Martin Grosell \\ University of Miami, \\ John D. Stieglitz \\ University of Miami, \\ John P Berry ( $\nabla$ berryj@fiu.edu ) \\ Florida International University https://orcid.org/0000-0002-3463-5560
}

\section{Research Article}

Keywords: Perfluoroalkyl substances (PFAS), fish, marine, mahi-mahi, embryotoxicity

Posted Date: February 7th, 2022

DOI: https://doi.org/10.21203/rs.3.rs-1293185/v1

License: (c) (i) This work is licensed under a Creative Commons Attribution 4.0 International License. Read Full License

Version of Record: A version of this preprint was published at Ecotoxicology on August 18th, 2022. See the published version at https://doi.org/10.1007/s10646-022-02576-w. 


\section{Abstract}

Perfluoroalkyl substances (PFAS) are highly persistent organic pollutants that have been detected in a wide array of environmental matrices and, in turn, biota including humans and wildlife wherein they have been associated with a multitude of toxic, and otherwise adverse effects, including ecosystem impacts. In the present study, we developed a toxicity assay for embryonic stages of mahi-mahi (Coryphaena hippurus), as an environmentally relevant pelagic fish species, and applied this assay to the evaluation of the toxicity of "legacy" and "next-generation" PFAS including, respectively, perfluorooctanoic acid (PFOA) and several perfluoroethercarboxylic acids (PFECA). Acute embryotoxicity, in the form of lethality, was measured for all five PFAS toward mahi-mahi embryos with median lethal concentrations $\left(\mathrm{LC}_{50}\right)$ in the micromolar range. Consistent with studies in other similar model systems, and specifically the zebrafish, embryotoxicity in mahi-mahi generally (1) correlated with fluoroalkyl chain length and hydrophobicity, i.e., log P, of PFAS, and thus, aligned with a role of uptake in the relative toxicity; and (2) increased with continuous exposure, suggesting a possible role of developmental stage specifically including a contribution of hatching (and loss of protective chorion) and/or differentiation of target systems (e.g., liver). Compared to prior studies in the zebrafish embryo model, mahi-mahi was significantly more sensitive to PFAS which may be related to differences in either exposure conditions (e.g., salinity) and uptake, or possibly differential susceptibility of relevant targets, for the two species. Moreover, when considered in the context of the previously reported concentration of PFAS within upper sea surface layers, and co-localization of buoyant eggs (i.e., embryos) and other early developmental stages (i.e., larvae, juveniles) of pelagic fish species to the sea surface, the observed toxicity potentially aligns with environmentally relevant concentrations in these marine systems. Thus, impacts on ecosystems including, in particular, population recruitment are a possibility. The present study is the first to demonstrate embryotoxicity of PFAS in a pelagic marine fish species, and suggests that mahi-mahi represents a potentially informative, and moreover, environmentally relevant, ecotoxicological model for PFAS in marine systems.

\section{Introduction}

Perfluoroalkyl substances (PFAS) are environmental contaminants of growing scientific, health and regulatory interest, having been detected ubiquitously in diverse environmental matrices, and consequently, biota including plants, wildlife and humans (Giesy and Kannan 2001; Prevedouros et al. 2006; Wang et al. 2013). Owing to their surfactant properties (derived from their amphiphilic nature), and high chemical stability (due to their fluorocarbon backbone), PFAS have been extensively used over the past nearly 80 years in household products, cosmetics, firefighting foams, food packaging materials, and as water repellants for various consumer products (Keml 2015; Wang et al. 2013). Due, however, to this same chemical stability and amphiphilicity, PFAS are both highly persistent and bioaccumulative, and therefore, have been found to be widespread in the environment (Ankley et al. 2021; McCarthy et al. 2017). As the historically most widely used and thus, both most environmentally prevalent, and best studied congeners, the so-called "legacy" PFAS including perfluorooctanoic acid (PFOA; Fig. 1) and perfluorooctane sulfonate (PFOS) have been shown to exhibit considerable environmental persistence and bioaccumulation potential. And their presence in the environment has been putatively linked to adverse effects on human and health including reproductive toxicity, carcinogenicity, teratogenicity and possible neurotoxicity, as well as other organ-specific toxicity including liver (i.e., hepatotoxicity) and kidney (i.e., nephrotoxicity), and possible links to metabolic syndrome (Lau et al. 2007; Pérez et al. 2013; Prevedouros et al. 2006; Shrestha et al. 2017). In addition to human health concerns, a growing body of evidence including documented bioconcentration and bioaccumulation, and ecotoxicological assessments, points to potential impacts of PFAS on ecosystems (Ankley et al. 2021; Burkhard 2021). 
Due to their prevalance in the environment, potential to bioaccumulate in biota, and associated health concern (Prevedouros et al. 2006; Wang et al. 2017), PFOA and PFAS have been largely phased-out from US and Europe, and replaced with several "next generation" alternatives. Most prominent of these alternatives are numerous perfluoroether carboxylic acids (PFECA; Fig. 1) that replace one or more fluorocarbon (-CF $\left.2^{-}\right)$in perfluoroalkyl chains with ether oxygens (-O-), and thereby, retain similar physicochemical (i.e., surfactant, stability) properties. Perhaps the most notable examples of PFECA, in current use, include the proprietary compounds GenX (i.e., perfluoro (2-methyl-3oxahexanoic)) and ADONA ${ }^{\mathrm{TM}}$ (i.e., dodecafluoro-3H-4,8-dioxanonanoate). Although PFECA have been introduced, as replacements to legacy PFAS, in response to environmental health concerns (Bowman 2015), several recent toxicological studies have suggested quantitatively similar or, in some cases, greater toxicity and/or potential for bioaccumulation (Gomis et al. 2015; Wang et al. 2015). And PFECA, therefore, potentially represent "emerging" environmental toxicants of concern.

Of particular concern with regards to PFAS is contamination of aquatic systems including groundwater, freshwater bodies and marine systems (Xiao et al. 2017). Contamination of waterways not only raises concerns for human health (e.g., drinking water, contamination of seafood), but also has potential consequences for aquatic ecosystems and species. Therefore, a number of recent toxicological studies have utilized various aquatic vertebrate (i.e., fish) and invertebrate species to investigate potential ecotoxicity of PFAS (Giesy et al. 2010; McCarthy et al. 2017). Of the studies to investigate PFAS toxicity, those employing the zebrafish (Danio rerio), a well-established laboratory model, including both adult (Jantzen et al. 2016) and early life (i.e., embryos, larvae) stages are particularly notable. The zebrafish embryo model, in particular, has been employed to evaluate a wide range of legacy (i.e., PFOA, PFOS) and next-generation PFAS including PFECA (Annunziato et al. 2019; Gebreab et al. 2020; Godfrey et al. 2017; Hagenaars et al. 2011; Jantzen et al. 2016; Menger et al. 2020; Pecquet et al. 2020; Shi et al. 2008; Wasel et al. 2021; Weiss-Errico et al. 2017; Ye et al. 2009; Zheng et al. 2012). While the zebrafish embryo model has been promoted as a general proxy for vertebrate toxicity, and as such, a possible model for human health (Bambino and Chu 2017; Bradford et al. 2017), toxicological studies in zebrafish, as a representative teleost fish species, also have potentially direct relevance to the impact of toxicants on aquatic biota. Toxicity studies of the early life (i.e., embryos, larvae) stages of fish, moreover, have specific ecotoxicologica/ relevance as toxicity with respect to these developmental stages reveal implications for population recruitment, and in turn, aquatic ecosystems as a whole.

This said, while prior studies with well-established laboratory animal models, such as the zebrafish, have provided important insight to the toxicity of PFAS, their value with respect to ecotoxicology is, however, arguably limited. Laboratory lines of zebrafish, for example, have minimal relevance as "ecological receptors" as both a species, in general, and moreover, as genetically non-wild type (i.e., laboratory bred) representatives of the species. At the same time, relatively few studies have assessed toxicity in marine species, compared to studies of freshwater species, and even fewer have focused on marine vertebrates. Indeed, a very recent and thorough review (Ankley et al. 2021) of the literature found that $<5 \%$ of published toxicology studies cited marine (versus freshwater) fish. And among the limited studies that have investigated "marine" fish, several have actually focused on anadromous rainbow trout (Oncorhynchyus mykiss (Palmer et al. 2002a; Robertson and Gaines 1986), and estuarine species of sheepshead minnow (Cyprinodon variegatus; (Palmer et al. 2002b) and medaka (Oryzias melastigma; (Fang et al. 2014; Huang et al. 2011). The few studies that have evaluated truly marine species (i.e., rockfish, eel, cod; (Ankley et al. 2021) have generally measured sub-acute physiological effects on adult stages.

Impacts on strictly marine (e.g., pelagic) fish, and moreover, early life stages of these species, may be especially relevant based on their natural history: while concentrations of PFAS in marine waters are typically low, and specifically below parts-per-trillion (ppt), recent studies have shown significant enrichment at or near the air-sea 
interface including, in particular, the sea surface microlayer (SSML) where buoyant, free-floating eggs (and embryos) of many marine fish species are localized. Ju et al. (2008), for example, reported an approximately 1.5 -fold increase of PFOS and PFOA in surface water $(<20 \mathrm{~cm})$ compared to subsurface water $(>30 \mathrm{~cm})$, but as much as a 100-fold or more enrichment within the SSML, compared to subsurface water. More recently, Casas et al. (2020) similarly reported enrichment of a wide range of PFAS in the surface layer of near-shore waters, and although enrichment factors for SSML were only up to 5-fold relative to seawater in this study, a nearly 5000-fold enrichment was observed for seawater aerosols, similarly suggesting a mechanism for surface concentration of PFAS.

To fill gaps in knowledge with respect to the ecotoxicology of PFAS in marine ecosystems, we developed an assay system based on early life (i.e., embryo) stages of the marine, ecologically, and commercially relevant fish species, mahi-mahi (Coryphaena hippurus). Mahi-mahi is a pelagic, high-trophic level, highly migratory species dispersed throughout the world's tropical and subtropical seas and oceans (Maggio et al. 2019; Palko et al. 1982; Perrichon et al. 2019), and highly valued in both sports fishing and commercial fisheries (Oxenford and Hunte 1999). Like many other pelagic marine fish, the species is characterized by both frequent spawning, and high fecundity (Beardsley Jr 1967; Maggio et al. 2019), with positively buoyant eggs localized to the surface of the water column (Perrichon et al. 2019), and embryos characterized by high growth and metabolic rates (Pasparakis et al. 2016). As an ecologically relevant species, early life stages of mahi-mahi have been recently developed as a model for environmental toxicology, and specifically demonstrated in relation to toxicity of crude oil fractions in association with oil spills in the Gulf of Mexico (Edmunds et al. 2015; Esbaugh et al. 2016; Heuer et al. 2019; Kirby et al. 2019; Nelson et al. 2016; Perrichon et al. 2018; Stieglitz et al. 2016). In the present study, we utilized assays based on the mahi-mahi embryo model to evaluate acute embryotoxicity of a representative sample (Fig. 1) of both legacy PFAS (i.e., PFOA), and several PFECA (Fig. 1) as emerging toxicants of concern. Embryotoxicity in mahi-mahi is compared, in turn, to our previously evaluated (Gebreab et al. 2020) toxicity of these same compounds in the zebrafish embryo model.

\section{Materials And Methods}

\section{Chemicals}

Perfluorooctanoic acid (PFOA, 96\% purity) was purchased from Sigma-Aldrich (St. Louis, MO, U.S.A.), and PFECA including perfluoro-3,6,9-trioxatridecanoic acid (PFO3TDA, 98\% purity), perfluoro-3,6-dioxadecanoic acid (PFO2DA, 97\% purity), 4-(heptafluoroisopropoxy) acid (PFDMMOBA) and perfluoro (2-methyl-3-oxahexanoic) acid (GenX, 97\% purity) were purchased from SynQuest Laboratories (Dallas, TX U.S.A.). All chemicals were utilized without further purification. Stock solutions of PFECAs and PFOA were prepared in bio-filtered seawater (i.e., hatchery system water; see Mahi-mahi rearing and breeding/spawning) in polypropropylene tubes (to prevent adsorption to glass(Shafique et al. 2017), and sonicated until complete dissolution of the compounds was achieved. Stock solutions were diluted in filtered seawater over a relevant range of concentrations for assessment of mahi-mahi embryo toxicity (see Mahimahi embryo toxicity assays).

\section{Mahi-mahi rearing and breeding/spawning}

Embryos of mahi-mahi (Coryphaena hippurus) were obtained from wild-caught mating pairs spawned in the University of Miami Experimental Hatchery (UMEH). The wild-caught mahi-mahi broodstock fish were collected from water offshore of Miami, Florida and were transported, acclimated, and spawned according to protocols detailed by Stieglitz et al. (2017). Broodstocks of mahi-mahi were maintained in two 15,000-L fiberglass tanks outfitted with filtered and UV-sterilized seawater inflows $\left(25-28^{\circ} \mathrm{C}\right)$, and fed daily with a mixture of chopped squid, mackerel, and sardines (Kloeblen et al. 2018; Stieglitz et al. 2017). Fertilized eggs (i.e., embryos) produced from the volitional 
spawning events were collected $4-6$ hours post-spawning, rinsed in UV-sterilized seawater, and transported in a cooler ( 30 min transit time) to FIU laboratories for exposure and toxicity testing. All breeding of mahi-mahi was conducted under protocols approved by the University of Miami Institutional Animal Care and Use Committee (IACUC18-052 LF).

\section{Mahi-mahi embryo toxicity assay}

An assay to evaluate embryotoxicity of PFAS toward mahi-mahi embryos was developed, specifically adapted from assay formats previously developed and validated for zebrafish embryotoxicity of PFAS (Berry et al. 2007; Gebreab et al. 2020; Weiss-Errico et al. 2017). Preliminary trials were conducted to establish a suitable exposure parameters including concentration range for each compound, and identified an effective percent weight (i.e., parts per million) concentration range of 1, 5, 10, 20, 30, 50, 100, 150, 200 and 250 ppm for all PFAS. Test solutions for exposures were prepared by dilution of sonicated stocks (in filtered seawater in polypropylene tubes), and $1 \mathrm{~mL}$ of each was transferred to corresponding wells of 24-well polypropylene test plates with 6 wells $(n=6)$ for each treatment concentration, along with 6 control wells (on each assay plate) of filtered seawater only. Each treatment was tested in triplicate. Prior to assays, embryos in stock plates (< $8 \mathrm{~h}$ post-spawning) were evaluated using a dissecting microscope, and all unhealthy (i.e., dead, moribund or deformed) embryos were removed; only clearly viable embryos were, thus, selected for assays. For assays, one embryo was distributed into each well of 24-well test plates, and assay plates were maintained within an environmental control chamber at $27^{\circ} \mathrm{C}$ and $16: 8$ dark/light for $48 \mathrm{~h}$. Plates were observed using a dissection microscope at 24 and 48 hours post-fertilization (hpf), and lethality (based on mobility, response to stimuli and presence/absence of heartbeat) recorded as an endpoint of toxicity. All toxicity assays were performed under protocols approved by the Florida International University's IACUC (IACUC-19-085), and by trained investigators.

\section{Data analysis}

Median lethal concentrations ( $\mathrm{LC}_{50}$ ), and their 95\% confidence intervals, for each (of 3 ) treatment replicates were calculated by Probit Analysis in SPSS (version 26.0; IBM Corporation Armonk, NY, USA, 2015), as well as for pooled data (from triplicate measurements). Lowest-observed-adverse-effect level (LOAEL) and no-observed-adverse-effect level (NOAEL) were calculated for pooled data (i.e., mean percent survival from triplicate measurements of each treatment) by comparison to untreated (negative) controls using a t-test. Sigmoidal fit for concentration-response curves, based on pooled data from triplicates, were performed using origin 2019b (Origin Lab, Northampton, Massachusetts, USA). One-way ANOVA, followed by Tukey's post hoc test, was used to calculate the statistical significance of the $\mathrm{LC}_{50}$ values with significance level of $\mathrm{p}<0.05$.

\section{Results And Discussion}

The current study is the first report of embryotoxicity of PFAS in a pelagic marine fish species. Dose-dependent embryotoxicity (Fig. 2), and specifically, lethality, was observed for mahi-mahi embryos exposed to all PFAS evaluated within $24 \mathrm{~h}$ of exposure in the micromolar range (Table 1). Measured toxicity in the mahi-mahi embryo generally aligns with previous studies in the zebrafish model which have reported quantitatively similar (i.e., micromolar) embryotoxicity for both legacy PFAS (i.e., PFOA and PFOS) and next-generation PFAS including PFECA (Gaballah et al. 2020; Gebreab et al. 2020; Godfrey et al. 2017; Hagenaars et al. 2011; Jantzen et al. 2016; Pecquet et al. 2020; Shi et al. 2008; Weiss-Errico et al. 2017; Ye et al. 2009; Zheng et al. 2012). Observations in both the present (i.e. mahimahi) and past (i.e., zebrafish) studies are notable as PFECA (e.g., GenX) have been adopted as purportedly less toxic alternatives (Bowman 2015), and these results, thereby, agree with prior studies which have concluded that toxicity of 
these replacement compounds is, on the contrary, potentially comparable to legacy PFAS (Gaballah et al. 2020; Gebreab et al. 2020).

Relative embryotoxicity for mahi-mahi, based on calculated 24-h $\mathrm{LC}_{50}$ values, was found to be GenX $<$ PFOA $<$ PFO2DA < PFDMMOBA < PFO3TDA, and was generally correlated with PFAS chain length (inclusive of all fluorocarbon and ether groups) with the notable exception of the relatively high toxicity of PFDMMOB. Indeed, LC $_{50}$ was significantly correlated ( $<$ < 0.001) when PFMMOBA was not included (Fig. 3 ). This trend - and, furthermore, the exception of the PFMMOBA - is noteworthy as a similar significant correlation between embryotoxicity and chain length of PFAS has been consistently demonstrated in numerous, previous studies (in the zebrafish embryo model; Buhrke et al. 2013; Gaballah et al. 2020; Menger et al. 2020; Ulhaq et al. 2013; Wasel et al. 2021) including a recent assessment of the same PFECA (Gebreab et al. 2020; see Table 1). These studies collectively demonstrate a highly reproducible, quantitative toxicity of PFAS in embryonic fish models. It has, likewise, been recently shown that bioconcentration factors (BCF) of PFAS (alongside relative toxicity) in zebrafish are, likewise, significantly correlated with chain length, and it has been concluded that toxic potential is likely correlated with relative uptake potential (Menger et al. 2020; Vogs et al. 2019). Moreover, a recent metabolomics analysis in the zebrafish embryo model (Gebreab et al. 2020) identified nearly identical patterns of altered metabolic profiles between embryos exposed to both PFOA, and long- and short-chain PFECA (i.e., PFO3TDA and GenX, respectively). This observation suggests shared mechanistic pathways of toxicity, despite observed quantitative differences, and correlations between embryotoxicity and chain-length, and thus, further support a role of differential uptake (rather than difference in mechanism) in the relative toxicity of perfluorocarboxylic acids. Relative uptake is presumably a function of hydrophobicity, and indeed, a similar correlation between toxicity and calculated log P values for PFAS (with similar exception of PFDMMOBA) was observed in the present study (Fig. 3). That said, while hydrophobicity and, in turn, uptake, of PFAS is expected to primarily increase with chain length, previous studies have suggested other structural features including, a role of terminal functional groups (e.g., carboxylic versus sulfonic acids) in the relative uptake and, thus, toxic potential (Menger et al. 2020; Vogs et al. 2019). Thus, correlations as observed here are likely to only apply to perfluorinated carboxylic acids.

Although the reason for the higher than expected toxicity of PFDMMOBA in the present study is unclear, and remains to be investigated further, it is worth noting that this congener is among the only two (with the other being the most lipophilic, long-chain PFO3TDA) for which LC $_{50}$ and LOAEL/NOAEL (Table 1) did not significantly change following an additional $24 \mathrm{~h}$ of exposure (discussed further below). And, in fact, a similar lack of significant increase in toxicity during an equivalent developmental window (7 dpf, i.e., fully hatched, early larval stage) was, likewise, observed for PFDMMOBA in a previous study of zebrafish embryotoxicity (Gebreab et al. 2020). It is, therefore, proposed that more rapid uptake, or alternatively perhaps, different cellular or molecular targets during early development, may explain higher than expected toxicity observed for this congener. Such a role of toxicokinetics and/or other mechanistic differences in the exceptional toxicity of this congener, however, remains to be addressed.

With the exception of PFDMMOBA, as well as most toxic PFO3TDA, lethality significantly increased for all compounds tested over $48 \mathrm{~h}$ of static exposure, as evidenced by significantly $(p<0.05)$ lower $L_{50}$, and NOAEL/LOAEL (Table 1). Similarly increased toxicity, based on both lethality and other toxicological endpoints, with continuous exposure has been observed in previous studies of PFAS including PFOA and PFECA in zebrafish embryos (Gebreab et al. 2020; Hagenaars et al. 2011; Ye et al. 2009; Zheng et al. 2012). With respect to developing embryos, increased toxicity at $48 \mathrm{~h}$ is notable as mahi-mahi embryos typically hatch within approximately 36-48 hpf (Perrichon et al. 2019), and nearly all (96\%) of the embryos in the current study were, in fact, fully hatched at $48 \mathrm{~h}$. Increased toxicity, therefore, may be associated with loss of the protective chorion as a barrier for uptake of PFAS. A 
role of the chorion would, in turn, have ecotoxicological implications for environmental exposure of post-hatch eleutheroembryos and larvae of mahi-mahi. A similarly significant increase in toxicity coincident with hatching $(\sim 72$ hpf) has previously been observed for the same compounds (except PFMDMMOBA) in zebrafish (Gebreab et al. 2020). A recent study (Vogs et al. 2019) has similarly documented a "biphasic" pattern of slower uptake prior to hatching, and accelerated uptake post-hatch, in the zebrafish embryo model generally concluding that the chorion, indeed, serves as a protective barrier. The current results are consistent with such a bi-phasic pattern, yet reflect differential effects of the chorion for PFAS congeners: specifically, rapid uptake (and little effect of chorion) of PFO3TDA and PFDMMOBA is seemingly reflected in the observation of a near maximum toxicity of these congeners within $24 \mathrm{~h}$, whereas the chorion limits uptake of PFOA, GenX and PFO2DA until hatching. Notably, the correlation between toxicity and chain-length is not maintained after $48 \mathrm{~h}$ exposure (Supplementary Fig. 1), and the differential role of the chorion may, furthermore, explain this lack of correlation: in short, as hindrance of the chorion does not exist post-hatch, equivalent uptake of congeners leads to a convergence of lethal concentrations (in the low micromolar range) after hatching.

Alternatively, it is possible that increased toxicity with exposure time might relate, at least in part, to the development of relevant cellular and molecular targets of PFAS. As recognized hepatotoxins, for example, both liver (i.e., hepatocytes) and enzymes associated with phase I hepatic detoxification including, in particular, cytochrome P450 have been well documented as targets of PFAS(Bassler et al. 2019; Cheng and Klaassen 2008; Dale et al. 2020). Studies in the zebrafish have recently suggested a role of the differentiation of the liver and hepatic enzymes in the observed stage-dependent increase in embryotoxicity of both PFOA and PFECA (Gebreab et al. 2020), as well as other hepatotoxins (Zuberi et al. 2019). Expression of genes associated with differentiation of hepatocytes, and associated liver enzymes, in mahi-mahi has been similarly found (Xu et al. 2017) to occur over a time-frame (i.e., 36 to $48 \mathrm{hpf}$ ) coincident with increased embryotoxicity in the current study, suggesting a possibly similar contribution of hepatic developmental in this toxicity. Elevated mortality may, of course, be simply due to the cumulative exposure to the compounds during the continuous exposure period. Whether the observed increased toxicity is due to the loss of the protective chorion barrier, development of the liver (or possibly other targets), or simply, to the prolonged duration (and cumulative effects) of exposure remains to be clarified.

Although observed toxicity of PFOA and PFECA in the mahi-mahi embryo system was approximately comparable in scale (i.e., $\mu \mathrm{M}$ concentrations) to that previously observed in the zebrafish model, and significantly correlated, with respect to the relative toxicity (with the exception of PFDMMOBA), between the two species (Fig. 3), comparison of the current data to our previous assessments of the same compounds in the zebrafish embryo model (Gebreab et al. 2020) demonstrated consistently higher toxicity, as evidenced by LC50 and LOAEL values (Table 1). The consensus of previous studies (Gaballah et al. 2020; Godfrey et al. 2017; Hagenaars et al. 2011; Pecquet et al. 2020; Ye et al. 2009; Zheng et al. 2012) of PFAS including PFOA and PFECA, e.g., GenX, in the zebrafish embryo model have, likewise, generally observed lower toxicity, i.e., LC $_{50}$, compared to that currently reported for mahi-mahi. Higher relative toxicity for mahi-mahi, compared to zebrafish, may be related to a number of factors including differences in toxicokinetics (i.e., uptake) and susceptibility of relevant biochemical, molecular or cellular targets between the two species, as well as respective assay parameters including, in particular, exposure media (e.g., seawater versus nonsaline medium, $\mathrm{pH}$, etc.). A positive correlation between salinity and BCF in fish has, for example, been previously demonstrated (Jeon et al. 2010), suggesting a possible role of both exposure medium (i.e., salinity), and consequent toxicokinetics, in the higher toxicity among mahi-mahi embryos. Alternatively, however, higher sensitivity of mahimahi may relate to the targeting of interrelated pathways of cellular energy metabolism by PFAS. Numerous previous studies have implicated metabolic dysfunction including lipid, amino acid and carbohydrate among the adverse effects of PFOA and PFECA (Alderete et al. 2019; Chen et al. 2020; Yu et al. 2016) including such effects in

Page $7 / 16$ 
early life (i.e., embryo and larval) stages of zebrafish (Gebreab et al. 2020; Sant et al. 2021). At the same time, it has been shown that rapidly developing mahi-mahi embryos are among the most metabolically active of marine fish species (Pasparakis et al. 2016), and much higher than zebrafish embryos. Taken together, it is possible that targeting of energy metabolism by PFAS may accentuate toxicity in mahi-mahi embryos, alongside any contributions of differential uptake and toxicokinetics.

Regardless of cause, the higher sensitivity of mahi-mahi may have implications for potential exposure of embryos to toxic concentrations of PFAS in ecologically relevant (i.e., marine) waters. Indeed, a lingering ecotoxicological question, in this regard, is whether environmentally relevant concentrations of PFAS in aquatic systems are sufficient for toxicity. With 48-h LOAEL (for lethality) in the low micromolar range for PFAS (Table 1), it is possible that subacute toxicity may, indeed, effectively extend into the nanomolar range. While typically in the sub-ppt range, PFAS concentrations approaching nanomolar concentrations (e.g., 200 ppt PFOA) have been measured, particularly in nearshore marine waters (Yamashita et al. 2004). Moreover, 100-fold concentration factors of PFAS within the SSML - and as much as 5000-fold in aerosols - have been reported (Casas et al. 2020; Ju et al. 2008), suggesting potentially nanomolar concentrations within ecologically relevant upper-layer surface waters where buoyant eggs (i.e., embryos) and larval stages of mahi-mahi, and many other marine fish species, are distributed. Whether these early life stages of marine fish are, in fact, exposed to effectively toxic concentrations, however, remains to be investigated.

In conclusion, embryos of mahi-mahi, as a representative pelagic marine fish species, were found to be a quantitative model of the toxicity of PFAS, largely comparable in this regard to the zebrafish embryo as an established laboratory model. Alongside quantitative potential of this model with respect to structure-activity (i.e., chain-length and relative toxicity), these studies point to interactive effects of uptake and developmental stage (e.g., hatching/loss of chorion, development of relevant target organs). Moreover, these studies identified toxicity at exposure concentrations sufficiently low to approach environmentally relevant concentrations, particularly in marine waters, and especially the sea surface where both PFAS and buoyant eggs of many marine fish species are generally concentrated. This finding opens the door to future studies to evaluate ecotoxicological impacts of PFAS on marine fish populations.

\section{Declarations}

Acknowledgements The authors would like to acknowledge support from Florida International University, University Graduate School's Presidential Fellowship program, which financially supported, in part, KG. MG is a Maytag Professor of Ichthyology.

\section{Funding}

Financial support for KG was provided by Florida International University Graduate School Presidential Fellowship program.

\section{Competing interests}

The authors declare they have no financials interests related to the research.

\section{Author contributions}

Mahi-mahi embryos used in the study were provided by JS, DB and MG from the UM Experimental Fish Hatchery. KG and JB contributed to the study conception and design. Data collection and analysis were performed by KG. The first 
draft of the manuscript was written by $K G$ and $J B$, and all authors commented on subsequent revisions of the manuscript. All authors read and approved the final manuscript.

\section{Ethics approval}

Hatchery spawning of mahi-mahi was conducted protocols approved by the University of Miami Institutional Animal Care and Use Committee (IACUC, 18-052-LF). All toxicity assays involving mahi-mahi were performed under protocols approved by the Florida International University IACUC (IACUC-19-085), and performed by trained investigators.

\section{Consent to participate}

Does not apply as no human subjects were involved in the research.

\section{Consent to publish}

Does not apply as no human subjects were involved in the research.

\section{References}

1. Alderete TL, Jin R, Walker DI, et al. (2019) Perfluoroalkyl substances, metabolomic profiling, and alterations in glucose homeostasis among overweight and obese Hispanic children: A proof-of-concept analysis. Environ Int 126:445-453.https://doi.org/10.1016/j.envint.2019.02.047

2. Ankley GT, Cureton P, Hoke RA, et al. (2021) Assessing the Ecological Risks of Per- and Polyfluoroalkyl Substances: Current State-of-the Science and a Proposed Path Forward. Environ Toxicol Chem 40(3):564605.https://doi.org/10.1002/etc.4869

3. Annunziato KM, Jantzen CE, Gronske MC, Cooper KR (2019) Subtle morphometric, behavioral and gene expression effects in larval zebrafish exposed to PFHxA, PFHxS and 6:2 FTOH. Aquat Toxicol 208:126137.https://doi.org/10.1016/j.aquatox.2019.01.009

4. Bambino K, Chu J (2017) Zebrafish in Toxicology and Environmental Health. Curr Top Dev Biol 124:331367.https://doi.org/10.1016/bs.ctdb.2016.10.007

5. Bassler J, Ducatman A, Elliott M, et al. (2019) Environmental perfluoroalkyl acid exposures are associated with liver disease characterized by apoptosis and altered serum adipocytokines. Environ Pollut 247:10551063.https://doi.org/10.1016/j.envpol.2019.01.064

6. Beardsley Jr GL (1967) Age, growth, and reproduction of the dolphin, Coryphaena hippurus, in the Straits of Florida. Copeia:441-451

7. Berry JP, Gantar M, Gibbs PD, Schmale MC (2007) The zebrafish (Danio rerio) embryo as a model system for identification and characterization of developmental toxins from marine and freshwater microalgae. Comp Biochem Physiol C Toxicol 145(1):61-72

8. Bowman JS (2015) Fluorotechnology is critical to modern life: the FluoroCouncil counterpoint to the Madrid Statement. Environ Health Perspect 123(5):A112-A113

9. Bradford YM, Toro S, Ramachandran S, et al. (2017) Zebrafish models of human disease: gaining insight into human disease at ZFIN. ILAR journal 58(1):4-16

10. Buhrke T, Kibellus A, Lampen A (2013) In vitro toxicological characterization of perfluorinated carboxylic acids with different carbon chain lengths. Toxicol Lett 218(2):97-104.https://doi.org/10.1016/j.toxlet.2013.01.025 
11. Burkhard LP (2021) Evaluation of published bioconcentration factor (BCF) and bioaccumulation factor (BAF) data for per-and polyfluoroalkyl substances across aquatic species. Environ Toxicol Chem

12. Casas G, Martinez-Varela A, Roscales JL, Vila-Costa M, Dachs J, Jimenez B (2020) Enrichment of perfluoroalkyl substances in the sea-surface microlayer and sea-spray aerosols in the Southern Ocean. Environ Pollut 267:115512.https://doi.org/10.1016/j.envpol.2020.115512

13. Chen Z, Yang T, Walker DI, et al. (2020) Dysregulated lipid and fatty acid metabolism link perfluoroalkyl substances exposure and impaired glucose metabolism in young adults. Environ Int 145:106091.https://doi.org/10.1016/j.envint.2020.106091

14. Cheng X, Klaassen CD (2008) Critical role of PPAR-a in perfluorooctanoic acid-and perfluorodecanoic acidinduced downregulation of Oatp uptake transporters in mouse livers. Toxicol Sci 106(1):37-45

15. Dale K, Yadetie F, Muller MB, et al. (2020) Proteomics and lipidomics analyses reveal modulation of lipid metabolism by perfluoroalkyl substances in liver of Atlantic cod (Gadus morhua). Aquat Toxicol 227:105590.https://doi.org/10.1016/j.aquatox.2020.105590

16. Edmunds RC, Gill JA, Baldwin DH, et al. (2015) Corresponding morphological and molecular indicators of crude oil toxicity to the developing hearts of mahi mahi. Sci Rep 5(1):17326.https://doi.org/10.1038/srep17326

17. Esbaugh AJ, Mager EM, Stieglitz JD, et al. (2016) The effects of weathering and chemical dispersion on Deepwater Horizon crude oil toxicity to mahi-mahi (Coryphaena hippurus) early life stages. Sci Total Environ 543(Pt A):644-651.https://doi.org/10.1016/j.scitotenv.2015.11.068

18. Fang S, Zhao S, Zhang Y, Zhong W, Zhu L (2014) Distribution of perfluoroalkyl substances (PFASs) with isomer analysis among the tissues of aquatic organisms in Taihu Lake, China. Environ Pollut 193:224-232

19. Gaballah S, Swank A, Sobus JR, et al. (2020) Evaluation of Developmental Toxicity, Developmental Neurotoxicity, and Tissue Dose in Zebrafish Exposed to GenX and Other PFAS. Environ Health Perspect 128(4):47005.https://doi.org/10.1289/EHP5843

20. Gebreab KY, Eeza MNH, Bai T, et al. (2020) Comparative toxicometabolomics of perfluorooctanoic acid (PFOA) and next-generation perfluoroalkyl substances. Environ Pollut 265(Pt

A):114928.https://doi.org/10.1016/j.envpol.2020.114928

21. Giesy JP, Kannan K (2001) Global distribution of perfluorooctane sulfonate in wildlife. Environ Sci Technol 35(7):1339-42.https://doi.org/10.1021/es001834k

22. Giesy JP, Naile JE, Khim JS, Jones PD, Newsted JL (2010) Aquatic toxicology of perfluorinated chemicals. Rev Environ Contam Toxicol 202:1-52.https://doi.org/10.1007/978-1-4419-1157-5_1

23. Godfrey A, Hooser B, Abdelmoneim A, Horzmann KA, Freemanc JL, Sepulveda MS (2017) Thyroid disrupting effects of halogenated and next generation chemicals on the swim bladder development of zebrafish. Aquat Toxicol 193:228-235.https://doi.org/10.1016/j.aquatox.2017.10.024

24. Gomis MI, Wang Z, Scheringer M, Cousins IT (2015) A modeling assessment of the physicochemical properties and environmental fate of emerging and novel per- and polyfluoroalkyl substances. Sci Total Environ 505:98191.https://doi.org/10.1016/j.scitotenv.2014.10.062

25. Hagenaars A, Vergauwen L, De Coen W, Knapen D (2011) Structure-activity relationship assessment of four perfluorinated chemicals using a prolonged zebrafish early life stage test. Chemosphere 82(5):764-772

26. Heuer RM, Galli GLJ, Shiels HA, et al. (2019) Impacts of Deepwater Horizon Crude Oil on Mahi-Mahi (Coryphaena hippurus) Heart Cell Function. Environ Sci Technol 53(16):9895-9904.https://doi.org/10.1021/acs.est.9b03798

27. Huang Q, Fang C, Wu X, Fan J, Dong S (2011) Perfluorooctane sulfonate impairs the cardiac development of a marine medaka (Oryzias melastigma). Aquat Toxicol 105(1-2):71- 
7.https://doi.org/10.1016/j.aquatox.2011.05.012

28. Jantzen CE, Annunziato KM, Cooper KR (2016) Behavioral, morphometric, and gene expression effects in adult zebrafish (Danio rerio) embryonically exposed to PFOA, PFOS, and PFNA. Aquat Toxicol 180:123130.https://doi.org/10.1016/j.aquatox.2016.09.011

29. Jeon J, Kannan K, Lim HK, Moon HB, Kim SD (2010) Bioconcentration of perfluorinated compounds in blackrock fish, Sebastes schlegeli, at different salinity levels. Environ Toxicol Chem 29(11):2529-2535

30. Ju X, Jin Y, Sasaki K, Saito N (2008) Perfluorinated surfactants in surface, subsurface water and microlayer from Dalian Coastal waters in China. Environ Sci Technol 42(10):3538-42.https://doi.org/10.1021/es703006d

31. Keml O (2015) use of highly 717 fluorinated substances and alternatives. Swedish Chemicals Agency

32. Kirby AR, Cox GK, Nelson D, et al. (2019) Acute crude oil exposure alters mitochondrial function and ADP affinity in cardiac muscle fibers of young adult Mahi-mahi (Coryphaena hippurus). Comp Biochem Physiol C Toxicol Pharmacol 218:88-95.https://doi.org/10.1016/j.cbpc.2019.01.004

33. Kloeblen S, Stieglitz JD, Suarez JA, Grosell M, Benetti DD (2018) Characterizing egg quality and larval performance from captive mahi-mahi Coryphaena hippurus (Linnaeus, 1758) spawns over time. Aquac Res 49(1):282-293

34. Lau C, Anitole K, Hodes C, Lai D, Pfahles-Hutchens A, Seed J (2007) Perfluoroalkyl acids: a review of monitoring and toxicological findings. Toxicol Sci 99(2):366-94.https://doi.org/10.1093/toxsci/kfm128

35. Maggio T, Allegra A, Andaloro F, et al. (2019) Historical separation and present-day structure of common dolphinfish (Coryphaena hippurus) populations in the Atlantic Ocean and Mediterranean Sea. ICES J Mar Sci 76(4):1028-1038

36. McCarthy C, Kappleman W, DiGuiseppi W (2017) Ecological considerations of per-and polyfluoroalkyl substances (PFAS). Curr Pollut Rep 3(4):289-301

37. Menger F, Pohl J, Ahrens L, Carlsson G, Orn S (2020) Behavioural effects and bioconcentration of per- and polyfluoroalkyl substances (PFASs) in zebrafish (Danio rerio) embryos. Chemosphere

245:125573.https://doi.org/10.1016/j.chemosphere.2019.125573

38. Nelson D, Heuer RM, Cox GK, et al. (2016) Effects of crude oil on in situ cardiac function in young adult mahimahi (Coryphaena hippurus). Aquat Toxicol 180:274-281

39. Oxenford HA, Hunte W (1999) Feeding habits of the dolphinfish (Coryphaena hippurus) in the eastern Caribbean. Scientia Marina 63(3-4):303-315

40. Palko BJ, Beardsley GL, Richards WJ (1982) Synopsis of the biological data on dolphin-fishes, Coryphaena hippurus Linnaeus and Coryphaena equiselis Linnaeus.

41. Palmer S, Van Hoven R, Krueger H (2002a) Perfluorooctanesulfonate, potassium salt (PFOS): A 96-hr static acute toxicity test with the rainbow trout (Oncorhynchus mykiss) Report No 454A-145 EPA Docket AR226-1030a044. Wildlife International Ltd. Easton, MD

42. Palmer S, Van Hoven R, Krueger H (2002b) Perfluorooctanesulfonate, potassium salt (PFOS): A 96-hr static renewal acute toxicity test with the sheepshead minnow (Cyprinodon variegatus). Wildlife International Ltd. Report No. 454A-146A

43. Pasparakis C, Mager EM, Stieglitz JD, Benetti D, Grosell M (2016) Effects of Deepwater Horizon crude oil exposure, temperature and developmental stage on oxygen consumption of embryonic and larval mahi-mahi (Coryphaena hippurus). Aquat Toxicol 181:113-123.https://doi.org/10.1016/j.aquatox.2016.10.022

44. Pecquet AM, Maier A, Kasper S, Sumanas S, Yadav J (2020) Exposure to perfluorooctanoic acid (PFOA) decreases neutrophil migration response to injury in zebrafish embryos. BMC Res Notes

Page 11/16 
13(1):408.https://doi.org/10.1186/s13104-020-05255-3

45. Pérez F, Nadal M, Navarro-Ortega A, et al. (2013) Accumulation of perfluoroalkyl substances in human tissues. Environ Int 59:354-362

46. Perrichon P, Mager EM, Pasparakis C, et al. (2018) Combined effects of elevated temperature and Deepwater Horizon oil exposure on the cardiac performance of larval mahi-mahi, Coryphaena hippurus. PLoS One 13(10):e0203949.https://doi.org/10.1371/journal.pone.0203949

47. Perrichon P, Stieglitz JD, Xu EG, et al. (2019) Mahi-mahi (Coryphaena hippurus) life development: morphological, physiological, behavioral and molecular phenotypes. Dev Dyn 248(5):337-350.https://doi.org/10.1002/dvdy.27

48. Prevedouros K, Cousins IT, Buck RC, Korzeniowski SH (2006) Sources, fate and transport of perfluorocarboxylates. Environ Sci Technol 40(1):32-44.https://doi.org/10.1021/es0512475

49. Robertson DR, Gaines SD (1986) Interference compoetition structures habitat use in a local assemblage of coral reef surgeonfishes. Ecology 67(5):1372-1383

50. Sant KE, Annunziato K, Conlin S, et al. (2021) Developmental exposures to perfluorooctanesulfonic acid (PFOS) impact embryonic nutrition, pancreatic morphology, and adiposity in the zebrafish, Danio rerio. Environ Pollut 275:116644.https://doi.org/10.1016/j.envpol.2021.116644

51. Shafique U, Schulze S, Slawik C, Kunz S, Paschke A, Schüürmann G (2017) Gas chromatographic determination of perfluorocarboxylic acids in aqueous samples-A tutorial review. Anal Chim Acta 949:8-22

52. Shi X, Du Y, Lam PK, Wu RS, Zhou B (2008) Developmental toxicity and alteration of gene expression in zebrafish embryos exposed to PFOS. Toxicol Appl Pharmacol 230(1):23-32.https://doi.org/10.1016/j.taap.2008.01.043

53. Shrestha S, Bloom MS, Yucel R, et al. (2017) Perfluoroalkyl substances, thyroid hormones, and neuropsychological status in older adults. Int J Hyg Environ Health 220(4):679-

685.https://doi.org/10.1016/j.jjheh.2016.12.013

54. Stieglitz JD, Hoenig RH, Kloeblen S, Tudela CE, Grosell M, Benetti DD (2017) Capture, transport, prophylaxis, acclimation, and continuous spawning of Mahi-mahi (Coryphaena hippurus) in captivity. Aquaculture 479:1-6

55. Stieglitz JD, Mager EM, Hoenig RH, Benetti DD, Grosell M (2016) Impacts of Deepwater Horizon crude oil exposure on adult mahi-mahi (Coryphaena hippurus) swim performance. Environ Toxicol Chem 35(10):26132622.https://doi.org/10.1002/etc.3436

56. Ulhaq M, Orn S, Carlsson G, Morrison DA, Norrgren L (2013) Locomotor behavior in zebrafish (Danio rerio) larvae exposed to perfluoroalkyl acids. Aquat Toxicol 144-145:332-40.https://doi.org/10.1016/j.aquatox.2013.10.021

57. Vogs C, Johanson G, Näslund M, et al. (2019) Toxicokinetics of perfluorinated alkyl acids influences their toxic potency in the zebrafish embryo (Danio rerio). Environ Sci Technol 53(7):3898-3907

58. Wang Z, Cousins IT, Scheringer M, Hungerbuehler K (2015) Hazard assessment of fluorinated alternatives to long-chain perfluoroalkyl acids (PFAAs) and their precursors: status quo, ongoing challenges and possible solutions. Environ Int 75:172-179

59. Wang Z, Cousins IT, Scheringer M, Hungerbühler K (2013) Fluorinated alternatives to long-chain perfluoroalkyl carboxylic acids (PFCAs), perfluoroalkane sulfonic acids (PFSAs) and their potential precursors. Environ Int 60:242-248

60. Wang Z, DeWitt JC, Higgins CP, Cousins IT (2017) A never-ending story of per-and polyfluoroalkyl substances (PFASs)? ACS Publications

61. Wasel O, Thompson KM, Gao Y, et al. (2021) Comparison of zebrafish in vitro and in vivo developmental toxicity assessments of perfluoroalkyl acids (PFAAs). J Toxicol Environ Health A 84(3):125-

136.https://doi.org/10.1080/15287394.2020.1842272

Page $12 / 16$ 
62. Weiss-Errico MJ, Berry JP, O'Shea KE (2017) beta-Cyclodextrin Attenuates Perfluorooctanoic Acid Toxicity in the Zebrafish Embryo Model. Toxics 5(4):31.https://doi.org/10.3390/toxics5040031

63. Xiao X, Ulrich BA, Chen B, Higgins CP (2017) Sorption of poly-and perfluoroalkyl substances (PFASs) relevant to aqueous film-forming foam (AFFF)-impacted groundwater by biochars and activated carbon. Environ Sci Technol 51(11):6342-6351

64. Xu EG, Mager EM, Grosell M, Hazard ES, Hardiman G, Schlenk D (2017) Novel transcriptome assembly and comparative toxicity pathway analysis in mahi-mahi (Coryphaena hippurus) embryos and larvae exposed to Deepwater Horizon oil. Sci Rep 7(1):44546.https://doi.org/10.1038/srep44546

65. Yamashita N, Kannan K, Taniyasu S, et al. (2004) Analysis of perfluorinated acids at parts-per-quadrillion levels in seawater using liquid chromatography-tandem mass spectrometry. Environ Sci Technol 38(21):5522-5528

66. Ye L, Wu LL, Jiang YX, Zhang CJ, Chen L (2009) [Toxicological study of PFOS/PFOA to zebrafish (Danio rerio) embryos]. Huan Jing Ke Xue 30(6):1727-32

67. Yu N, Wei S, Li M, et al. (2016) Effects of Perfluorooctanoic Acid on Metabolic Profiles in Brain and Liver of Mouse Revealed by a High-throughput Targeted Metabolomics Approach. Sci Rep 6(1):23963.https://doi.org/10.1038/srep23963

68. Zheng X-M, Liu H-L, Shi W, Wei S, Giesy JP, Yu H-X (2012) Effects of perfluorinated compounds on development of zebrafish embryos. Environ Sci Pollut Res 19(7):2498-2505

69. Zuberi Z, Eeza MNH, Matysik J, Berry JP, Alia A (2019) NMR-Based Metabolic Profiles of Intact Zebrafish Embryos Exposed to Aflatoxin B1 Recapitulates Hepatotoxicity and Supports Possible Neurotoxicity. Toxins (Basel) 11(5):258.https://doi.org/10.3390/toxins11050258

\section{Table}

Table 1. Relevant measures of embryotoxicity including median lethal concentration ( LC $\left._{50}\right)$, no-observed-adverseeffect level (NOAEL) and lowest-observed-adverse-effect level (LOAEL), and comparison of median lethal concentrations to zebrafish embryo model $\left(\mathrm{ZF} \mathrm{LC}_{50}\right)$.

\begin{tabular}{|c|c|c|c|c|c|c|c|c|}
\hline \multirow{3}{*}{ Compound } & \multirow{3}{*}{$\begin{array}{l}\mathrm{LC}_{50} \\
(\mu \mathrm{M}) \\
{[95 \% \mathrm{Cl}]}\end{array}$} & \multicolumn{2}{|l|}{$24 \mathrm{hpf}$} & \multirow[b]{2}{*}{$\begin{array}{l}\mathrm{LC}_{50} \\
(\mu \mathrm{M})\end{array}$} & \multicolumn{2}{|l|}{$\begin{array}{l}48 \mathrm{hpf} \\
\mathrm{a}\end{array}$} & \multicolumn{2}{|c|}{$\begin{array}{l}\mathrm{ZF} \mathrm{LC}{ }_{50}(\mu \mathrm{M})[95 \% \\
\mathrm{Cl}^{\mathrm{a}, \mathrm{b}}\end{array}$} \\
\hline & & $\begin{array}{l}\text { NOAEL } \\
(\mu \mathrm{M})\end{array}$ & $\begin{array}{l}\text { LOAEL } \\
(\mu \mathrm{M})\end{array}$ & & $\begin{array}{l}\text { NOAEL } \\
(\mu \mathrm{M})\end{array}$ & $\begin{array}{l}\text { LOAEL } \\
(\mu \mathrm{M})\end{array}$ & \multirow{2}{*}{$24 \mathrm{hpf}$} & \multirow[t]{2}{*}{$7 \mathrm{dpf}$} \\
\hline & & & & {$[95 \% \mathrm{Cl}]$} & & & & \\
\hline PFOA & $\begin{array}{l}132 \\
{[98,176]}\end{array}$ & 24 & 48 & $9[4,15]^{\star}$ & 2 & 12 & $\begin{array}{l}232 \\
{[200,268]}\end{array}$ & $\begin{array}{l}122 \\
{[105,143]^{\star}}\end{array}$ \\
\hline GenX & $\begin{array}{l}253 \\
{[194,322]}\end{array}$ & - & 91 & $\begin{array}{l}59 \\
{[34,94]^{\star}}\end{array}$ & - & 91 & $\begin{array}{l}386 \\
{[331,446]}\end{array}$ & $\begin{array}{l}256 \\
\underset{\star}{[219,300]}\end{array}$ \\
\hline PFDMMOBA & $\begin{array}{l}44 \\
{[23,74]}\end{array}$ & 13 & 26 & $\begin{array}{l}26 \\
{[10,50]}\end{array}$ & 13 & 26 & $\begin{array}{l}249 \\
{[214,289]}\end{array}$ & $\begin{array}{l}211 \\
{[180,249]}\end{array}$ \\
\hline PFO2DA & $\begin{array}{l}76 \\
{[33,154]}\end{array}$ & 11 & 22 & $\begin{array}{l}2.6 \\
{[0.5,6.2]^{*}}\end{array}$ & - & 2 & $\begin{array}{l}159 \\
{[139,184]}\end{array}$ & $\begin{array}{l}25 \\
{[17,34]^{\star}}\end{array}$ \\
\hline PF03TDA & $\begin{array}{l}34 \\
{[16,63]}\end{array}$ & 9 & 18 & $12[7,19]$ & 2 & 9 & $40[32,47]$ & $\begin{array}{l}18 \\
{[12,25]^{\star}}\end{array}$ \\
\hline
\end{tabular}


a Statistical significance of difference between $\mathrm{LC}_{50}$ at 24 and $48 \mathrm{hpf}$ for mahi-mahi, and $24 \mathrm{hpf}$ and $7 \mathrm{dpf}$ for zebrafish, based on $95 \%$ confidence interval $(p<0.05)$, is indicated by asterisk $(*)$

b Zebrafish LC $_{50}$ values from Gebreab et al. (2020).

\section{Figures}<smiles>O=C(O)C(F)(F)C(F)(F)C(F)(F)C(F)(F)C(F)(F)C(F)(F)C(F)(F)F</smiles>

\section{PFOA}<smiles>O=C(O)C(F)(OC(F)(F)C(F)(F)C(F)(F)F)C(F)(F)F</smiles>

\section{GenX}<smiles>O=C(O)C(F)(F)C(F)(F)C(F)(F)OC(F)(F)C(F)(F)F</smiles>

\section{PFDMMOBA}<smiles>O=C(O)C(F)(F)OC(F)(F)C(F)(F)OC(F)(F)C(F)(F)C(F)(F)C(F)(F)F</smiles>

\section{PFO2DA}<smiles>O=C(O)C(F)(F)OC(F)(F)C(F)(F)OC(F)(F)C(F)(F)OC(F)(F)C(F)(F)C(F)(F)C(F)(F)F</smiles>

\section{PFO3TDA}

Figure 1

Perfluoralkyl substances investigated in the current study, including PFOA (A) and the perfluoroether carboxylic acids, GenX (B), PFDMMOBA (C), PFO2DA (D) and PFO3TDA (E). 

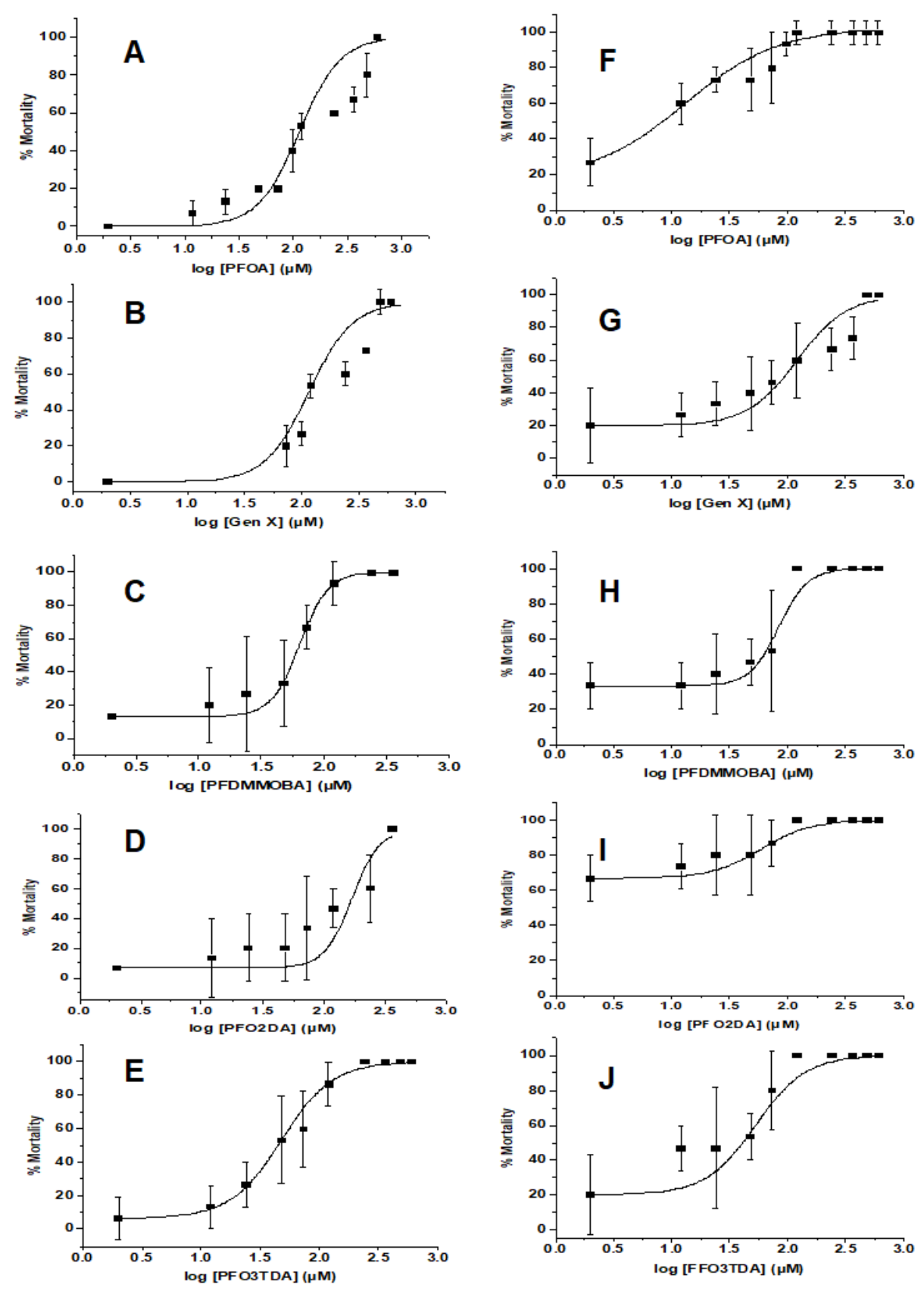

\section{Figure 2}

Concentration-dependent toxicity of PFOA, Gen X, PFDMMOBA, PFO2DA and PFO3TDA at 24 hpf (A-E, respectively) and $48 \mathrm{hpf}$ (F-J, respectively). Mean percent mortality pooled for each concentration from triplicate measurements. Error bar represents \pm standard deviation $(n=3)$ 

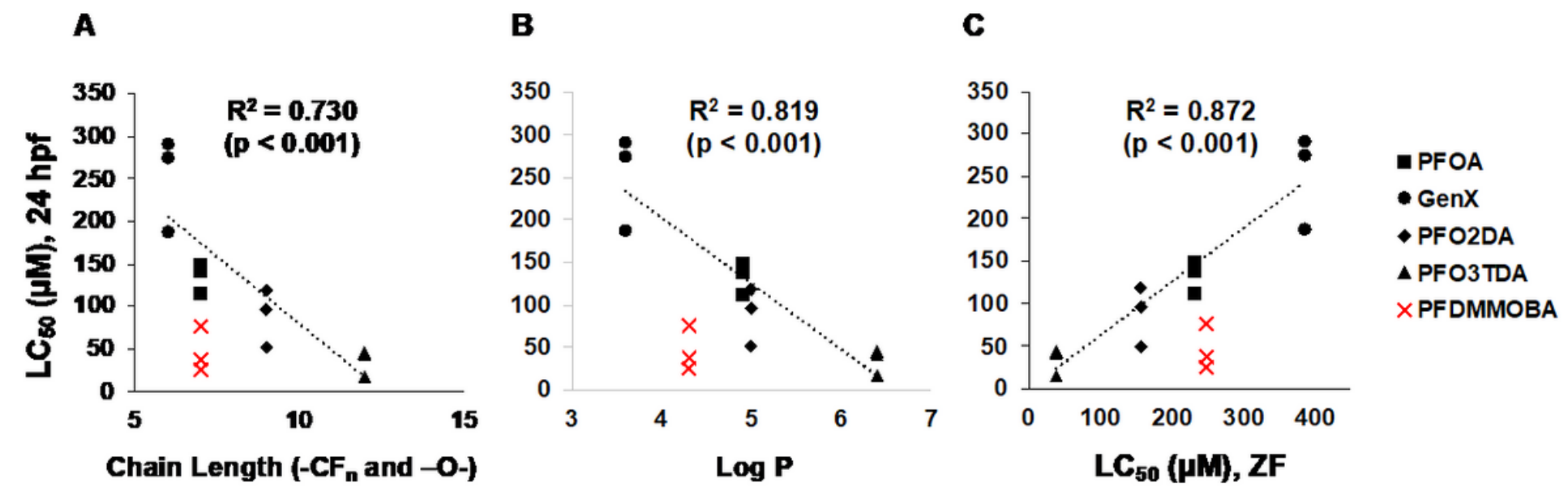

Figure 3

Correlation between mahi-mahi 24-h embryotoxicity, as measured by median lethal concentration ( $\left.\mathrm{LC}_{50}\right)$, and $(\mathrm{A})$ PFAS fluoroetheralkyl chain-length, (B) log P, and (C) embryotoxicity, i.e., 24-h LC 50 , previously reported for zebrafish (Gebreab et al. 2020). Log P values from National Center for Biotechnology Information

(https://pubchem.ncbi.nlm.nih.gov/, retrieved June 22, 2021). Chain-length inclusive of all fluorocarbons, ethers and terminal carboxylic acid group.

\section{Supplementary Files}

This is a list of supplementary files associated with this preprint. Click to download.

- SupplementaryInformationSuppFig1Gebreabetal.docx 